\title{
Implementation of Image Reconstruction for GE SIGNA PET/MR PET Data in the STIR Library
}

\author{
Palak Wadhwa, Student Member, IEEE, Kris Thielemans, Senior Member, IEEE, Nikos Efthimiou, Member, IEEE, \\ Ottavia Bertolli, Elise Emond, Benjamin A. Thomas, Michel Tohme, Kristen A. Wangerin, Gaspar Delso, William \\ Hallett, Member, IEEE, Roger N. Gunn, David Buckley, and Charalampos Tsoumpas, Senior Member, IEEE
}

\begin{abstract}
Software for Tomographic Image Reconstruction (STIR: http://stir.sf.net) is an open source $\mathrm{C}++$ library available for reconstruction of emission tomography data. This work aims at the incorporation of the GE SIGNA PET/MR scanner in STIR and enables PET image reconstruction with data corrections. The data extracted from the scanner after an acquisition includes a list of raw data files (emission, normalisation, geometric and well counter calibration (wcc) factors), magnetic resonance attenuation correction (MRAC) images and the scanner-based reconstructions. The listmode (LM) file stores a list of 'prompt' events and the singles per crystal per second. MRAC images from the scanner are used for attenuation correction. The modifications to STIR that allow accurate histogramming of this LM data in the same sinogram organisation as the scanner are also described. This allows reconstruction of acquisition data with all data corrections using STIR, and independent of any software supplied by the manufacturer. The implementations were validated by comparing the histogrammed data, data corrections and final reconstruction using the ordered subset expectation maximisation (OSEM) algorithm with the equivalents from the GE-toolbox, supplied by the manufacturer for the scanner. There is no difference in the histogrammed counts whereas an overall relative difference of $6.7 \times 10^{-8} \%$ and from $0.01 \%$ to $\mathbf{0 . 8 6 \%}$ is seen in the normalisation and randoms correction sinograms respectively. The STIR reconstructed images have similar resolution and quantification but have some residual differences due to wcc factors, decay and deadtime corrections, as well as the offset between PET and MR gantries that will be addressed in future work. This work will enable the use of all current and future STIR algorithms, including penalized image reconstruction, motion correction and direct parametric image estimation, on data from GE SIGNA PET/MR scanners.
\end{abstract}

\section{INTRODUCTION}

P. Wadhwa (email: p.wadhwa@leeds.ac.uk), D. Buckley and C. Tsoumpas are with Department of Biomedical Imaging Science, University of Leeds, UK.

P. Wadhwa, W. Hallett, R. N. Gunn and C.Tsoumpas are with Invicro, UK

K. Thielemans, O. Bertolli, E. Emond and B A. Thomas are with Institute of Nuclear Medicine, University College London, UK.

N. Efthimiou is with PET Research Centre, Faculty of Health Sciences, University of Hull, Hull HU6 7RX, UK.

M. Tohme and K A. Wangerin are with GE Healthcare, Waukesha, WI, US G. Delso is with GE Healthcare, Cambridge. UK

P. Wadhwa is funded by the Medical Research Council (MR/M01746X/1). C. Tsoumpas is sponsored by a Royal Society Industry Fellowship (IF170011). This project has been supported by EPSRC Collaborative Computational Flagship Project (EP/M022587/1 and EP/P022200/1).

We would like to thank Dr. Floris Jansen (GE Healthcare) and Nicholas Keat (Invicro) for their substantial support on progressing this project.

Ethics number 17/WM/0084 with permission from a clinical study performed at Invicro.
OFTWARE for tomographic image reconstruction (STIR) $\checkmark$ is a library build with $\mathrm{C}++$ that is capable of reconstructing emission tomography images for datasets acquired using different scanners [1]. This current work aims to extend the STIR library to be able to handle the acquired data from GE SIGNA PET/MR scanner. The scanner's time of flight (ToF) capabilities are not currently implemented in this work. The present modifications to the software are not trivial and therefore need to be assessed carefully using phantom experiments. This paper is divided into sections that include: (A) incorporation of the scanner geometric information in STIR; (B) native data format reading of acquisition and correction datasets; (C) phantom data acquisition; (D) reconstruction of acquired phantom datasets; (E) analysis of the images reconstructed from STIR in comparison to the GE-provided reconstruction software or "GE-toolbox", as it will be called in this summary hereafter.

\section{Materials \& Methods}

\section{A. GE SIGNA PET/MR Scanner Geometric Information}

The GE SIGNA PET/MR scanner is comprised of an integrated PET scanner located within a 3T MRI scanner [2]. The PET scanner has a patient bore radius of $31.2 \mathrm{~cm}$, the transaxial field of view (FOV) of $60 \mathrm{~cm}$ and an axial FOV of $25 \mathrm{~cm}$. The scanner has 45 rings with 448 detectors per ring. The scanner parameters are stored in the scanner template class.

\section{B. Acquisition and Data Correction Implementations}

- Reading the GE SIGNA PET/MR listmode Data: The LM data read from an uncompressed listmode (LM) Hierarchical Data Format (HDF5) file (extracted from the scanner) has been implemented by O. Bertolli. LM coincidence events are stored in a HDF5 file along with their corresponding crystal identifiers (IDs).

- Histogramming the LM data into STIR projection data: Im_to_projdata utility was used to convert the LM data to STIR projection data. This scanner stores sinograms as having span 3 in segment 0 and span 1 for all the other segments where span $\mathrm{N}$ refers to axial data from $\mathrm{N}$ ring differences combined into one axial plane [3]. Detector IDs run in the opposite transaxial directions for STIR and scanner due to different conventions. This conventional discrepancy is implemented in STIR for accurate histogramming. 
- Reading the uncompressed sinogram or 'rdf':

The scanner stores the 'prompts' or emission data as a series of 4D ToF viewgram. There are 224 such viewgrams each of dimensions $1981 \times 27 \times 357$, with 1981 axial positions, $27 \mathrm{ToF}$ bins and 357 tangential positions. In current implementations, ToF data is added together to get non-ToF viewgrams.

- Reading Singles Data from LM File:

In GE scanners, random events are estimated using single rates [4]. These have lower noise levels than the delayed window method. The formula that estimates randoms from singles per crystal is $R_{x y}=2 \tau S_{x} S_{y} / T$, where $R_{x y}$ is the number of random events (or counts) detected by the detector pair $x$ and $y, \tau$ is the coincidence time window, $T$ is the total acquisition time, $S_{x}$ and $S_{y}$ are the single events detected by crystal $x$ and $y$, respectively. The singles rates per second are read from the uncompressed LM HDF5 file.

- Reading Normalisation Data:

Normalisation HDF5 data files are extracted from PET raw data DICOM files. The 'norm3d' HDF5 file contains crystal efficiencies and the 'geo3d' HDF5 file contains the geometric correction viewgrams. The geometric and crystal efficiency factors are multiplied to get the normalisation correction factors per detector bin as $n_{i j}=\epsilon_{i} \epsilon_{j} g_{i j}$, where $n_{i j}$ are the normalisation correction factor for detector pairs $i$ and $j, \epsilon_{i}$ (respectively, $\epsilon_{j}$ ) are the crystal efficiency factors for detectors $i$ (respectively, $j$ ), and $g_{i j}$ is the geometric correction factor. Deadtime correction has not yet been implemented [5].

- Detector positioning:

Crystal 0 has a transaxial offset of $-5.23^{\circ}$ (negative sign represents anti-clockwise direction) contrary to the conventional $0^{\circ}$ adopted in STIR. We changed the STIR projector to take this offset into account.

\section{GE SIGNA PET/MR Data Acquisition}

For this study, three phantom and one patient datasets were acquired using the GE SIGNA PET/MR scanner.

1) Volumetric Quality Control (VQC) Phantom: The VQC phantom consists of five small ${ }^{68} \mathrm{Ge}$ spheres or VQC sources as it will be called in this summary, embedded in MR-visible tubes. These are low radiation spheres with $0.7 \mathrm{MBq}$ in each sphere, everything encased within a foam cube. The scan was conducted over 10 minutes resulting in $5 \times 10^{6}$ prompts.

2) Bottle Phantom: This phantom is a modified National Electrical Manufacturers Association (NEMA) Image quality (IQ) which consists of 6 fillable spheres with diameters varying from $10 \mathrm{~mm}$ to $37 \mathrm{~mm}$ filled with 5.38 $\mathrm{MBq}$ of $\left[{ }^{18} \mathrm{~F}\right]-\mathrm{FDG}$ [6]. A high activity bottle filled with $77.9 \mathrm{MBq}$ of $\left[{ }^{18} \mathrm{~F}\right]-\mathrm{FDG}$ was placed touching the two of the smallest spheres of the phantom. The phantom was scanned for 5 minutes resulting in $5 \times 10^{8}$ prompts.

3) Hoffmann Phantom [7]: The scan was conducted for 20 minutes resulting in $1.5 \times 10^{8}$ prompts.

4) Patient Dataset: A patient with fibrosis of the lung was injected with $40.62 \mathrm{MBq}$ of an experimental ${ }^{18} \mathrm{~F}$

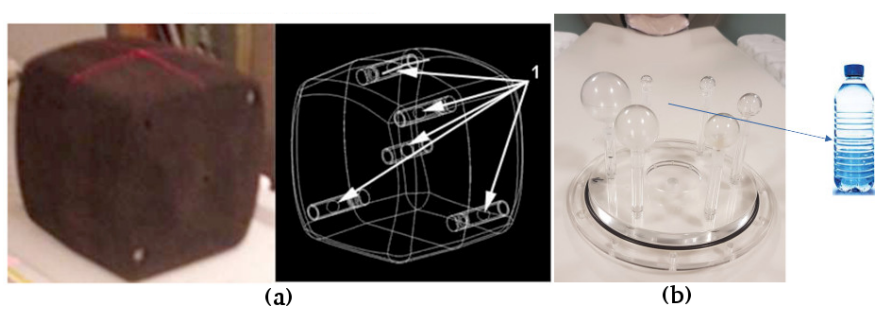

Fig. 1: (a) VQC Phantom with five small ${ }^{68} \mathrm{Ge}$ spheres and (b) Bladder Mimicking Bottle Phantom which consists of 6 fillable spheres and a $500 \mathrm{ml}$ bottle placed between smallest spheres.

radiotracer, 90 minutes prior to the scan. The scan duration was over 13 minutes resulting in $3.8 \times 10^{7}$ prompts.

MRAC images, PET images, uncompressed PET LM and PET raw data series were collected for the above scans.

\section{Image Reconstruction}

This work was carried out by histogramming LM data and estimating randoms, normalisation corrections using STIR utilities developed during this work. Attenuation correction was carried out using the GE provided PET Image For Attenuation (PIFA). The data was reconstructed using the OSEM algorithm with 28 subsets over 3 iterations. Results were compared to those obtained with the GE-toolbox reconstructions using VUE-point HD (GE Healthcare) algorithm with 28 subsets over 3 iterations. The images were reconstructed in a $305 \times$ $305 \times 89$ matrix, with a voxel size of $2.02 \times 2.02 \times 2.78 \mathrm{~mm}^{3}$.

\section{E. Data Analysis}

LM data histogrammed into STIR and GE-toolbox projection data were compared by subtracting the datasets. The comparisons were also carried out between the uncompressed sinograms that were read using STIR and the GE-toolbox projection data. The same comparison method was repeated for normalisation and randoms sinograms. The reconstructed images using STIR and the GE-toolbox were compared for the VQC phantom using a full-width-half-maximum (FWHM) calculation. This was done by drawing line profiles through the peak of two of the VQC sources at positions [172,188,34] $\mathrm{mm}$ and $[-17,0,34] \mathrm{mm}$ with respect to the center of the PET scanner. A Gaussian was fitted to the line profiles to estimate the FWHM.

\section{RESULTS \& DISCUSSION}

Figure 2 shows the LM data histogrammed into projection data by STIR and the GE-toolbox, normalisation and random sinograms obtained by STIR and the GE-toolbox and their relative differences. The comparisons were made after a single post-processing step which involved inverting the view and tangential positions of the GE-toolbox sinogram in MATLAB (as the GE-toolbox uses a different file format). There were no differences in the total counts that were histogrammed with STIR and the GE-toolbox. These sinograms were also compared with the non-ToF sinogram that was read from the 


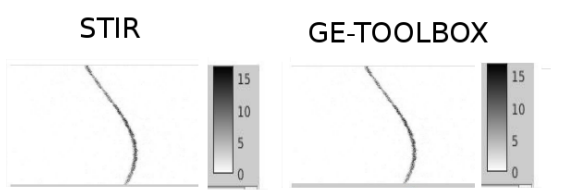

EMISSION

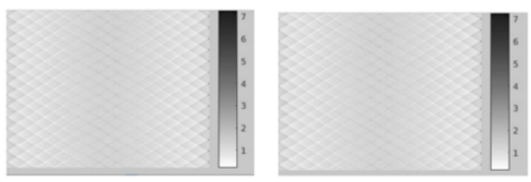

NORMALISATION

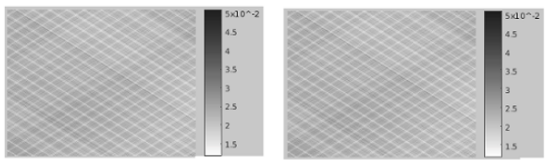

RANDOMS

Fig. 2: Sinogram Comparison: STIR and GE-TOOLBOX; The relative difference: for emission is $0 \%$, for normalisation is $6.7 \times 10^{-8} \%$ and for randoms is from $0.01 \%$ to $0.86 \%$.

TABLE I: Calculated FWHM for VQC sources as shown in Figure 3; along radial, tangential and axial directions. VQC source 1 and 2 are positioned at $[172,188,34]$ and $[-17,0,34] \mathrm{mm}$ respectively with respect to the center of the PET scanner.

\begin{tabular}{c|c|c|c}
\hline VCQ Source & Direction & STIR $(\mathbf{m m})$ & Toolbox $(\mathbf{m m})$ \\
\hline \hline & Radial & $19.4 \pm 1.8$ & $19.2 \pm 1.8$ \\
Source 1 & Tangential & $17.7 \pm 2.2$ & $19.6 \pm 1.8$ \\
& Axial & $18.5 \pm 1.6$ & $15.1 \pm 0.9$ \\
\hline \multirow{3}{*}{ Source 2 } & Radial & $19.4 \pm 0.5$ & $19.2 \pm 1.8$ \\
& Tangential & $16.3 \pm 0.4$ & $18.1 \pm 1.1$ \\
& Axial & $16.7 \pm 0.4$ & $18.7 \pm 0.6$ \\
\hline \hline
\end{tabular}

uncompressed 'rdf' extracted from the scanner. The STIR emission sinograms extracted either by histogramming or reading from 'rdf' were identical.

The randoms correction sinogram calculated by STIR and the GE-toolbox were compared and a relative difference from $0.01 \%$ to $0.86 \%$ was obtained. This is likely due to the advanced processing of decay and dead-time correction by the GE-toolbox. The normalisation sinogram also compared well and an overall relative difference of $6.7 \times 10^{-8} \%$ was obtained. Figure 3 shows the reconstructed images between STIR and the GE-toolbox. The visible translational offsets between STIR and the GE-toolbox reconstructions that can be seen in the VQC phantom images are because of residual alignment issues that are under investigation. The alignment differences correspond to measured VQC $x, y$ and $z$ translational offsets and a rotation between the PET and MR gantries. Alignment issues affecting attenuation correction are evident as well in the bottle phantom. Table I summarises the FWHM comparison of the VQC sources along radial, tangential and axial directions. The space within MR-visible tubes which encapsulates VQC spheres is measured to be $20.16 \mathrm{~mm}$. This development is expected to be included in the new release of STIR library.

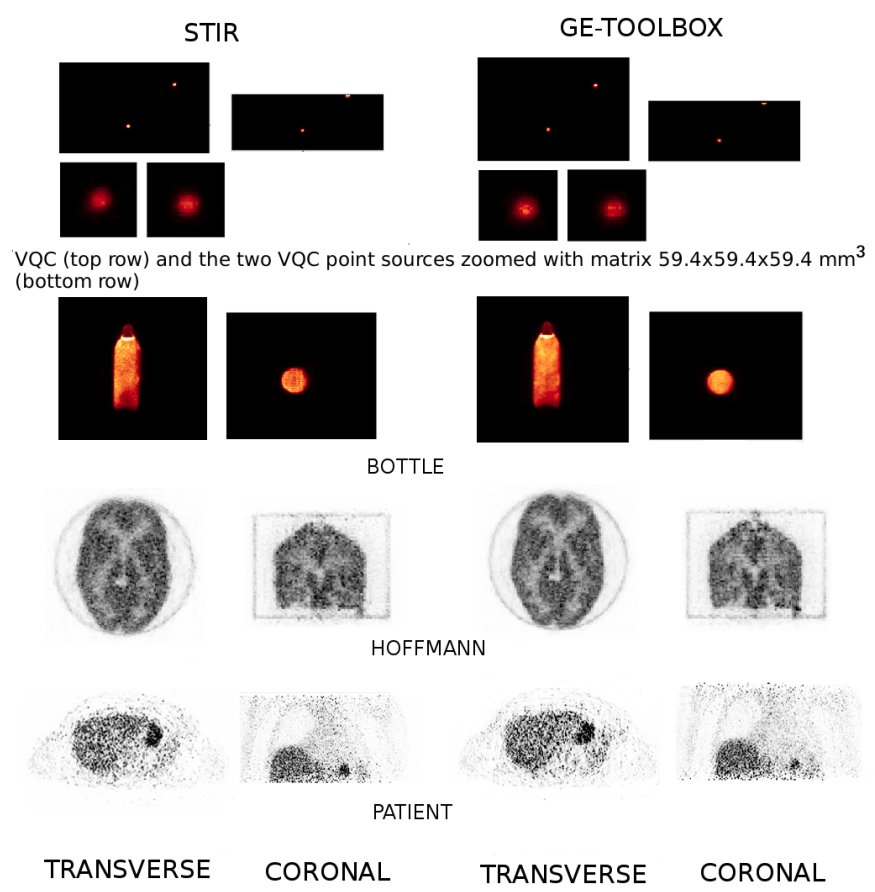

Fig. 3: Image Comparison: STIR and GE-TOOLBOX

\section{CONCLUSIONS}

In this study, we report the modifications needed to read data from the GE SIGNA PET/MR data directly in to STIR. This study shows successful reconstructions but with some apparent residual alignment offsets. We developed a framework for native GE SIGNA PET/MR data reconstruction in STIR. Here we use OSEM, additional iterative methods can be used due to the modular nature of STIR. Future work will incorporate time-of-flight (ToF) sinograms and allow ToF reconstruction with $\mathrm{ToF}$ scatter correction, implement dead-time correction and VQC offset within the reconstruction [8].

\section{REFERENCES}

[1] K. Thielemans, C. Tsoumpas, S. Mustafovic, T. Beisel, P. Aguiar, N. Dikaios, and M. W. Jacobson, "STIR: Software for Tomographic Image Reconstruction Release 2," Phys Med Biol, vol. 57, pp. 867-883, 2012.

[2] A. M. Grant, T. W. Deller, M. M. Khalighi, S. H. Maramraju, G. Delso, and C. S. Levin, "NEMA NU 2-2012 performance studies for the SiPMbased ToF-PET component of the GE SIGNA PET/MR system," Med Phys, vol. 43, pp. 2334-2343, 2016.

[3] F. H. Fahey, "Data acquisition in PET imaging," J Nucl Med Technol, vol. 30, pp. 39-49, 2002.

[4] C. W. Stearns, D. L. McDaniel, S. G. Kohlmyer, P. R. Arul, B. P. Geiser, and V. Shanmugam, "Random coincidence estimation from single event rates on the Discovery ST PET/CT scanner," vol. 5, p. 30673069, 2003.

[5] M. Defrise, D. E. Townsend, D. Bailey, A. Geissbuhler, C. Michel, and T. Jones, "A normalization technique for 3D PET data," Phys Med Biol, vol. 36, pp. 939 - 952, 1991.

[6] R. Boellaard, I. Rausch, T. Beyer, G. Delso, M. Yaqub, and H. H. Quick, "Quality control for quantitative multicenter whole-body PET/MR studies: a NEMA image quality phantom study with three current PET/MR systems," Med Phys, vol. 42, p. 59615969, 2015.

[7] E. Hoffman, P. Cutler, W. Digby, and J. Mazziotta, "3-D phantom to simulate cerebral blood flow and metabolic images for PET," IEEE Trans Nucl Sci, vol. 37, pp. 616-620, 1990.

[8] N. Efthimiou, E. Emond, P. Wadhwa, C. Cawthorne, C. Tsoumpas, and K. Thielemans, "Implementation and validation of time-of-flight PET image reconstruction module for listmode and sinogram projection data in the STIR library," Phys Med Biol, 2019: In Press. 